\title{
SOLVING IRREGULAR STRIP PACKING PROBLEMS WITH FREE ROTATIONS USING SEPARATION LINES
}

\author{
Jeinny Peralta $^{1^{*}}$, Marina Andretta ${ }^{1}$ and José Fernando Oliveira ${ }^{2}$
}

Received June 29, 2017 / Accepted March 17, 2018

\begin{abstract}
Solving nesting problems or irregular strip packing problems is to position polygons on a fixed width and unlimited length strip, obeying polygon integrity containment constraints and nonoverlapping constraints, in order to minimize the used length of the strip. To ensure non-overlapping, we use separation lines, i.e., straight lines that separate polygons. We present a nonlinear programming model that considers free rotations of the polygons and of the separation lines. This model uses a considerable smaller number of variables than the few other approaches proposed in the literature. We use the nonlinear programming solver IPOPT (an algorithm of interior points type), which is part of COIN-OR. Computational tests were run using established benchmark instances and the results were compared with the ones obtained with other methodologies in the literature that use free rotations.
\end{abstract}

Keywords: separation line, irregular packing problems, nonlinear optimization.

\section{INTRODUCTION}

Irregular strip packing problems have a great relevance in production processes, such as garment, manufacturing and furniture making. In irregular strip packing, smaller irregular pieces (in our case polygons) must be positioned into a big piece (in our case the strip), minimizing the used length of the strip. The main constraint in irregular strip packing problems is the non-overlapping between pieces, but it is very complex for a computational program to determine if two pieces are overlapping, touching or separated. In the literature there are tools for solving this issue (see [5]), among these the raster methods, direct trigonometry, no-fit polygon, and phi-function. In raster methods the strip is always divided into discrete areas and coding schemes are used. In the coding schemes used by Oliveira and Ferreira, and Segenreich and Braga in [22] and [25], respectively, the empty cells belonging to the division of the strip are encoded by zero and numbers equal to

\footnotetext{
*Corresponding author.

${ }^{1}$ Instituto de Ciências Matemáticas e de Computação, Universidade de São Paulo, Av.Trabalhador São-carlense, 400, 13566-590 São Carlos, SP, Brasil. E-mails: jeinnyp@icmc.usp.br; andretta@icmc.usp.br

2 INESC TEC, Faculdade de Engenharia, Universidade do Porto, Rua Dr. Roberto Frias, 4200-590 Porto, Portugal.

E-mail: jfo@fe.up.pt
} 
or greater than one are used to encode a piece; so, to check the non-overlap in raster methods is only a matter of checking the grid cells. There are several tools that use trigonometry to deal with the non-overlapping. In [14] circles inscribed are used to relax the non-overlapping constraints, replacing them with non-overlapping constraints of circles inscribed. In the remaining tools that use trigonometry, the pieces are represented by polygons, thereby the non-intersection of the edges of polygons must be checked to verify the non-overlap. In phi-function, the pieces are represented by the union or intersection of primary objects, that is, circles, rectangles, regular polygons, convex polygons, and the complement of these forms. This tool was designed and implemented in $[6,26,27,29]$. The phi-function is a mathematical expression that represents the relative position of two pieces. Specifically, the phi-function value is greater than zero if the pieces are separated; equal to zero if their borders are touching; and smaller than zero if overlapping each other. In this article, we use two tools to ensure non-overlapping of pieces, direct trigonometry and no-fit polygon. In the model, we represent the pieces by polygons and use separation lines, that is, we use trigonometry. A straight line is a separation line if given two polygons, all vertices of one of the polygons are on one side of the line or on the line, and all vertices of the other polygon are on the other side of the line or on the line. For the resolution of the modeled problem we need a starting solution; for the construction of this starting solution, we use the no-fit polygon. The no-fit polygon is a polygon resulting from the two polygons that are being compared. One of the advantages of this method is that the generation of these no-fit polygons is done only once, in a pre-processing phase, but a big disadvantage is that the no-fit polygons are dependent on the orientation of the polygons, and have to be generated for all their possible orientations; in all the instances, we use four predefined orientations of the polygons, $0^{\circ}, 90^{\circ}, 180^{\circ}$ and $270^{\circ}$, which is usually done in the literature.

Several solving techniques for these problems that deal with irregular pieces have been developed, based predominantly on heuristics and metaheuristics [1,10,13,17, 20,22]. The heuristics used for solving these problems can work with partial solutions, constructing the final layout piece by piece (constructive heuristics), or complete solutions, in which changes are done in order to find improvements. Exact algorithms based on mixed integer linear programming models that ensure finding the optimal solution were also developed $[2,12,31]$; however, in these algorithms, the runtime increases dramatically with the increase of the quantity of objects used in the problem. Few methods that combine exact and heuristic approaches have been developed [9], which allow the computation of high quality solutions in shorter computational times. In all these techniques and algorithms, free rotations are not allowed.

Additionally in the literature, we also find a visual system for packing problem of irregular pieces with free rotation into a rectangular board that aims to minimize the waste [16]. This algorithm is based on Physics, the rubber band physics movement.

Nonlinear programming models have also been proposed for representing the irregular packing problem, such as $[8,14,15,23,29]$. In all these models free rotation of the pieces is allowed. In [8] a model for a strip packing problem was presented. In this model phi-functions are used to ensure non-overlapping of the pieces. The pieces in this paper are phi-objects, which are 
$2 \mathrm{D}$ and $3 \mathrm{D}$ objects of very general type. To solve the problem [8] applied a modification of the Zoutendijk method of feasible directions [34,35] combined with the concept of $\epsilon$-active inequalities [28]. In [14] the pieces and the shapes can be arbitrary nonconvex polygons and to solve the problem [14] used three solvers: Branch\&Reduce Optimization Navigator (BARON) [24, 30]; the LINDO global optimization procedure (GOP); and The Global Mixed-Integer Quadratic Optimizer (GloMIQO) [18]. In [23], like in [8], a model for an irregular strip packing problem was presented. In this, the resolution of the problem is divided in two phases: big pieces are compacted in a first phase, while in a second phase, the remaining small pieces are placed between the big pieces. In their experiments, [23] used instances where the pieces are convex and nonconvex irregular polygons. The representation of these polygons was done by circle covering, and they used the nonlinear solver ALGENCAN [3,4]. In [15] a model for two cases of packing pieces was developed. In the first case, the objective is to pack the pieces in such a way as to minimize the area of the design rectangle. In the second case, the objective is to pack the pieces on stocked rectangles of known geometric dimensions. Separation lines are used to ensure non-overlapping. In their work the pieces are circles, rectangles, and convex polygons; and to solve the problem [15] used BARON [24,30] and LindoGlobal; an experiment was performed with only two polygons and found a feasible solution for it, in which LindoGlobal proved global optimality in $40 \mathrm{~min}$, but BARON did not increase the lower bound at all. With more than two polygons this technique has difficulty finding an optimal solution to the problem. In [29] it was provided a nonlinear programming model that employs ready-to-use phi-functions. In this paper, the pieces are bounded by circular arcs and/or line segments, and two types of container are considered, rectangular and circular. To solve the problem, [29] developed a compaction algorithm to search for local optimal solutions, which is performed by IPOPT (an algorithm of interior points type, [32]), a component of COIN-OR.

In this paper we propose a nonlinear mathematical model for an irregular strip packing problem which deals only with polygons which may be convex or nonconvex, and that can rotate freely. In the model, to ensure non-overlapping, we use direct trigonometry, in particular separation lines, a similar technique to that used in [15], but with a significantly lower number of variables. The high number of variables in the model used in [15] comes from having all vertices of the polygon as variables. Also, it is due to the line vector equation used to model the separation lines, which in turn implies the employment of many variables such as footing point vector, direction vector, normal vector, vectors connecting the separation line with the vertices, and distance of the vertices to the separation line, among others. In our model only the reference point and the angle of rotation for each polygon and for each separation line are variables, allowing us to obtain good solutions for larger instances in reasonable execution times. Like the polygons, the separation lines also can rotate freely. We use a code for nonlinear programming to solve the problem, IPOPT [32], which depends substantially on a starting solution. We present a way of calculating starting solutions.

This paper is organized as follows. In the next section a model of an irregular strip packing problem that considers free rotations is presented. The modeled problem, the polygons representation used in the model, and the tool used to ensure non-overlapping are described also in this section. 
In Section 3, the parameters of the algorithm used for solving the problem are presented, as well as the numerical results obtained when performing tests with known benchmark instances. At the end of our paper we present some conclusions in Section 4.

\section{A MODEL FOR AN IRREGULAR STRIP PACKING PROBLEM}

The irregular strip packing problem studied in this paper consists of placing $n$ irregular polygons, which can rotate freely, in a fixed width and unlimited length strip, obeying polygon integrity containment constraints and non-overlapping constraints, in order to minimize the used length of the strip. We propose a nonlinear mathematical model for this irregular strip packing problem.

We now explain how the polygons are represented in the model (Section 2.1), as well as the tool used to ensure non-overlapping of the polygons (Section 2.2), and then, introduce the model (Section 2.3).

\subsection{Representation of polygons in the model}

Here, we describe the representation of the polygons. If a polygon is convex, it is represented by their vertices, as follows:

$$
P_{i}=\left[\left(x_{i}^{1}, y_{i}^{1}\right),\left(x_{i}^{2}, y_{i}^{2}\right), \ldots,\left(x_{i}^{v_{i}}, y_{i}^{v_{i}}\right)\right],
$$

with $v_{i}$ the number of vertices of the polygon $P_{i}$.

If a polygon is nonconvex, it can be partitioned in convex polygons, as follows:

$$
P_{i}=\left[P_{i, 1}, P_{i, 2}, \ldots, P_{i, p_{i}}\right],
$$

with $p_{i}$ the number of convex polygons belonging to the partition of the nonconvex polygon $P_{i}$, see Figure 1.

The partition of a nonconvex polygon influences the model and therefore the solution, because there is a directly proportional relationship between the number of convex polygons belonging to the partition and the number of separation lines. The number of variables and the computational effort will grow according to the number of polygons in the partition.

The coordinates of a vertex belonging to a partition of a nonconvex polygon $P_{i}$ are given by

$$
\left(x_{i, j}^{l}, y_{i, j}^{l}\right), \text { with } j=1, \ldots, p_{i} \text { and } l=1, \ldots, v_{i, j},
$$

with $v_{i, j}$ the number of vertices of the convex polygon $P_{i, j}$.

We can deal with the problem with $n$ nonconvex polygons in the same way that we deal with the problem with $N$ convex polygons, with $N=\sum_{i=1}^{n} p_{i}$. We just have to ensure that the translations and rotations are the same for all polygons belonging to the partition of a nonconvex polygon.

The reference point is used for representing a polygon which has undergone translations and/or rotations, since we can write all other vertices of the polygon in terms of this point, as can be seen in Section 2.1.1. We choose the first vertex as the reference point of a polygon. 


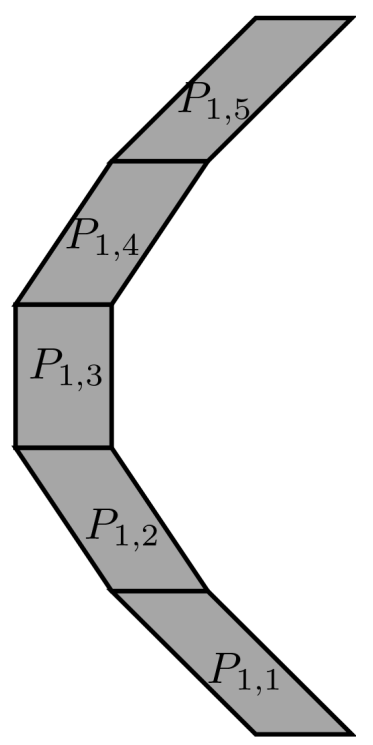

Figure 1 - Partition of a nonconvex polygon.

\subsubsection{Vertices of polygons in general form}

Henceforth, we will use the following notation: $\left(x_{i}^{l}, y_{i}^{l}\right)$ are the coordinates of a vertex of a polygon $P_{i}$ in the original position, and $\left(\bar{x}_{i}^{l}, \bar{y}_{i}^{l}\right)$ are the coordinates of a vertex of a polygon $P_{i}$ which has undergone translations and/or rotations.

In the representation of the polygons used in the model, we assume that $\left(x_{i}^{1}, y_{i}^{1}\right)=(0,0)$ for all $i$. Thus, if we translate and rotate a polygon $P_{i}$ around its reference point, the coordinates of any vertex $l$ in general form are given by:

$$
\left(\bar{x}_{i}^{l}, \bar{y}_{i}^{l}\right)=\left(x_{i}^{l} \cos \theta_{i}-y_{i}^{l} \sin \theta_{i}+\bar{x}_{i}^{1}, x_{i}^{l} \sin \theta_{i}+y_{i}^{l} \cos \theta_{i}+\bar{y}_{i}^{1}\right),
$$

with $\theta_{i}$ the angle of rotation of the polygon $P_{i}$. We consider that positive angles represent rotation in the counterclockwise direction.

When we deal with nonconvex polygons, we make sure that the translations and rotations are the same for all polygons belonging to the partition of the nonconvex polygon.

\subsection{Separation lines}

We use separation lines to ensure non-overlapping, see Figure 2. A straight line given by the equation $y=c_{i, r} x+d_{i, r}$ separates two polygons $P_{i}$ and $P_{r}$ if either

$$
\begin{cases}y_{i}^{l}-c_{i, r} x_{i}^{l}-d_{i, r} \leq 0, & \forall l \in\left\{1, \ldots, v_{i}\right\}, \\ y_{r}^{l}-c_{i, r} x_{r}^{l}-d_{i, r} \geq 0, & \forall l \in\left\{1, \ldots, v_{r}\right\}\end{cases}
$$


or

$$
\left\{\begin{aligned}
y_{i}^{l}-c_{i, r} x_{i}^{l}-d_{i, r} \geq 0, & \forall l \in\left\{1, \ldots, v_{i}\right\}, \\
y_{r}^{l}-c_{i, r} x_{r}^{l}-d_{i, r} \leq 0, & \forall l \in\left\{1, \ldots, v_{r}\right\},
\end{aligned}\right.
$$

holds.

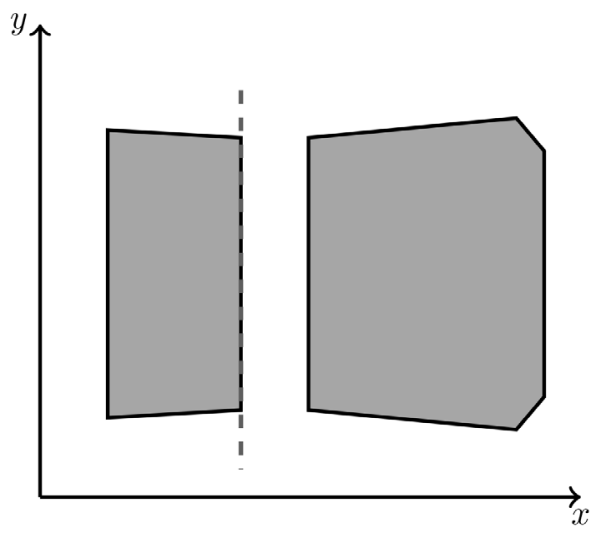

Figure 2 - Separation line of two convex polygons.

When we are dealing with nonconvex polygons, we must have lines separating each pair of polygons $P_{i, j}, P_{r, s}$, belonging to the partition of polygons $P_{i}, P_{r}$, respectively, with $i \neq r$ (that is, we do not have lines separating the polygons belonging to the partition of a nonconvex polygon), $j \in 1, \ldots, p_{i}$, and $s \in 1, \ldots, p_{r}$.

A separation line does not necessarily pass over one side of one of the two polygons that it is separating; however, at the starting solution, all separation lines have a rotation of $0^{\circ}$ and pass over one side of one of the two polygons that it is separating. Next, we present the general form of a separation line passing through a side of one of the two polygons, that is, which passes through two vertices of a polygon $\left(P_{i, j}\right.$ or $\left.P_{r, s}\right)$, let's say $\left(x_{k}, y_{k}\right)$ and $\left(x_{k+1}, y_{k+1}\right)$ :

$$
\left(y-y_{k}\right)\left(x_{k+1}-x_{k}\right)-\left(y_{k+1}-y_{k}\right)\left(x-x_{k}\right)=0 .
$$

Like polygons, the separation lines can also rotate and translate, as long as they remain being separation lines. When rotating and translating a separation line, we rewrite the point $\left(\bar{x}_{k+1}, \bar{y}_{k+1}\right)$ as a function of $\left(\bar{x}_{k}, \bar{y}_{k}\right)$, which we will call from now on reference point of the separation line; therefore

$$
\begin{aligned}
\left(\bar{x}_{k+1}, \bar{y}_{k+1}\right)= & \left(\left(x_{k+1}-x_{k}\right) \cos \alpha_{i, j, r, s}+\left(y_{k}-y_{k+1}\right) \sin \alpha_{i, j, r, s}+\bar{x}_{k},\right. \\
& \left.\left(x_{k+1}-x_{k}\right) \sin \alpha_{i, j, r, s}+\left(y_{k+1}-y_{k}\right) \cos \alpha_{i, j, r, s}+\bar{y}_{k}\right),
\end{aligned}
$$

with $\alpha_{i, j, r, s}$ the angle of rotation of the separation line of the polygons $P_{i, j}$ and $P_{r, s}$.

Next, we rewrite the separation line equation:

$$
\begin{aligned}
& \left(y-\bar{y}_{k}\right)\left[\left(x_{k+1}-x_{k}\right) \cos \alpha_{i, j, r, s}+\left(y_{k}-y_{k+1}\right) \sin \alpha_{i, j, r, s}\right]- \\
& \left(x-\bar{x}_{k}\right)\left[\left(x_{k+1}-x_{k}\right) \sin \alpha_{i, j, r, s}+\left(y_{k+1}-y_{k}\right) \cos \alpha_{i, j, r, s}\right]=0 .
\end{aligned}
$$




\subsection{Model for an irregular strip packing problem with $n$ polygons considering free rotations}

Because we want to position $n$ polygons in a fixed width and unlimited length strip in order to minimize the used length of the strip, the objective function is given by:

$$
z=\max \left\{\bar{x}_{i_{l}}\right\}, \quad l=1,2, \ldots, v_{i} \text { and } i=1,2, \ldots, n,
$$

with $v_{i}$ the number of vertices of the polygon $P_{i}$.

Without loss of generality, to ensure non-overlapping, we used in our model the set of constraints (1), for each pair of polygons $P_{i, j}$ and $P_{r, s}$, with

$$
c_{i, j, r, s}=\frac{\left(x_{k+1}-x_{k}\right) \sin \alpha_{i, j, r, s}+\left(y_{k+1}-y_{k}\right) \cos \alpha_{i, j, r, s}}{\left(x_{k+1}-x_{k}\right) \cos \alpha_{i, j, r, s}+\left(y_{k}-y_{k+1}\right) \sin \alpha_{i, j, r, s}}
$$

and

$$
d_{i, j, r, s}=\bar{y}_{k}-c_{i, j, r, s} \bar{x}_{k},
$$

in which $\left(x_{k}, y_{k}\right)$ and $\left(x_{k+1}, y_{k+1}\right)$ are the two vertices of one of the polygons whereby passes the separation line and $\alpha_{i, j, r, s}$ is the rotation angle of the straight line that separates $P_{i, j}$ from $P_{r, s}$. We use the notation $\left(x_{k}, y_{k}\right)$ for the reference point of the separation line. Note that the coordinates of this reference point are also the values of the translations parameters, that from now on, we will write $\left(\bar{x}_{i, j, r, s}, \bar{y}_{i, j, r, s}\right)$, for a straight line that is separating $P_{i, j}$ from $P_{r, s}$.

Let $\left(z, q_{1}, q_{2}, \ldots, q_{n}, \bar{q}_{1}, \bar{q}_{2}, \ldots, \bar{q}_{Q}\right)$ be the vector of all variables in our model, with $z$ the length of the strip defined in (3), $q_{i}$ the variables referring to the polygon $P_{i}, q_{i}=\left(\bar{x}_{i}^{1}, \bar{y}_{i}^{1}, \theta_{i}\right)$, $i=1, \ldots, n$ and $\bar{q}_{\ell}$ the variables referring to the line that separates polygon $P_{i, j}$ from polygon $P_{r, s}, \bar{q}_{\ell}=\left(\bar{x}_{i, j, r, s}, \bar{y}_{i, j, r, s}, \alpha_{i, j, r, s}\right), \ell=1, \ldots, Q$ and $Q=\sum_{i=1}^{n-1} p_{i}\left(\sum_{k=i+1}^{n} p_{k}\right)$ the number of separation lines. Let $e$ be the width of the strip in which the polygons are to be placed. A general model for our problem is given by:

Minimize $z$

$$
\text { subject to } 0 \leq \bar{y}_{i, j}^{l} \leq e, \quad \begin{array}{rl}
i & =1, \ldots, n \\
j & =1, \ldots, p_{i} \\
l & =1, \ldots, v_{i, j} \\
i & =1, \ldots, n \\
j & =1, \ldots, p_{i} \\
l & =1, \ldots, v_{i, j} \\
0 \leq \bar{x}_{i, j}^{l} \leq z, & , i=1, \ldots, n \\
r & =1, \ldots, n \\
i & \neq r \\
\bar{y}_{i, j}^{l}-c_{i, j, r, s} \bar{x}_{i, j}^{l}-d_{i, j, r, s} \leq 0 & j=1, \ldots, p_{i}
\end{array}
$$




$$
\begin{aligned}
s & =1, \ldots, p_{r} \\
l & =1, \ldots, v_{i, j} \\
\bar{y}_{r, s}^{l}-c_{i, j, r, s} \bar{x}_{r, s}^{l}-d_{i, j, r, s} \geq 0, i & =1, \ldots, n \\
r & =1, \ldots, n \\
i & \neq r \\
j & =1, \ldots, p_{i} \\
s & =1, \ldots, p_{r} \\
l & =1, \ldots, v_{r, s}
\end{aligned}
$$

Remembering that the vertices of a translated and rotated polygon $P_{i, j}$ are given by $\left(\bar{x}_{i, j}^{l}, \bar{y}_{i, j}^{l}\right)$, for $i=1, \ldots, n, j=1, \ldots, p_{i}$ and $l=1, \ldots, v_{i, j}$, in which

$$
\bar{x}_{i, j}^{l}=x_{i, j}^{l} \cos \theta_{i}-y_{i, j}^{l} \sin \theta_{i}+\bar{x}_{i}^{1},
$$

and

$$
\bar{y}_{i, j}^{l}=x_{i, j}^{l} \sin \theta_{i}+y_{i, j}^{l} \cos \theta_{i}+\bar{y}_{i}^{1},
$$

with $\left(\bar{x}_{i}^{1}, \bar{y}_{i}^{1}\right)$ the variable reference point of polygon $P_{i}$ and $\theta_{i}$ the variable rotation angle. $\left(x_{i, j}^{l}, y_{i, j}^{l}\right)$ are the coordinates of a vertex of a polygon $P_{i}$ in the original position.

Constraints (4a) and (4b) ensure that a polygon $P_{i}$ is entirely inside the strip. In these constraints, the width $e$ is a fixed parameter; the length $z$ is a variable; $\bar{x}_{i, j}^{l}$ and $\bar{y}_{i, j}^{l}$ depend on the reference point and the rotation angle of the polygon, $\left(\bar{x}_{i}^{1}, \bar{y}_{i}^{1}\right)$ and $\theta_{i}$, respectively, which are variables. Constraints (4c) and (4d) ensure non-overlapping of the convex polygons $P_{i, j}$ and $P_{r, s}$. In these constraints, $c_{i, j, r, s}$ and $d_{i, j, r, s}$ depend on the reference point and the rotation angle of the separation line, $\left(\bar{x}_{i, j, r, s}, \bar{y}_{i, j, r, s}\right)$ and $\alpha_{i, j, r, s}$, respectively, which are variables.

\section{COMPUTATIONAL EXPERIMENTS AND RESULTS}

All numerical experiments were performed on an Intel Core I7-4510U CPU @ 2.1GHz processor and $8 \mathrm{~GB}$ of memory. We used a code for nonlinear programming to solve the problem, IPOPT [32] (an algorithm of interior points type), which is part of the COIN-OR [33].

IPOPT is the implementation of a barrier or interior points method for large scale nonlinear optimization problems of the type

$$
\begin{array}{lc}
\text { Minimize } & f(v) \\
\text { subject to } & g^{L} \leq \\
& g(v) \leq g^{U}, \\
& v^{L} \leq v \leq v^{U},
\end{array}
$$

with $v \in \mathbb{R}^{n}$ and $f, g$ continuously differentiable; the mathematical details of this algorithm can be found in $[21,32]$. IPOPT can be executed in different programming languages, we used the 
C programming language version of Ipopt-3.12.3 and compiled the codes in the Ubuntu 12.04 operating system.

The CPU time is very large when we use the Hessian of the Lagrangian, therefore we will always use an option given in IPOPT to approximate the Hessian with limited memory, which makes the runtime shorter, without affecting the quality of the solution. In addition to the standard IPOPT parameters, we use the adaptive update strategy for barrier parameter. The maximum execution time is set to one hour.

We use the geometric library Computational Geometry Algorithms Library (CGAL) to partition the nonconvex polygons, in particular, we use the implementation of Greene's dynamic programming algorithm [7].

In the next subsection a brief explanation of the starting solution used in the execution of IPOPT is presented. In Section 3.2, the results obtained with IPOPT and comparisons of those with two methodologies recent in the literature [16,29], which also allow free rotations, are presented.

\subsection{Starting solutions}

IPOPT is designed to find local solutions. Taking into account that the developed nonlinear model is nonconvex, when solving it we can find many stationary points with different objective function values, and these stationary points depend on the starting solution.

In the next section we present the instances that are used to test our model. For each one of these instances, we generate a starting solution using a bottom-left algorithm. The bottom-left algorithm is a single pass heuristic that, given a set of pieces and an order, places the pieces one by one on the strip, as far to the left and to the bottom as possible. The algorithm receives a list of randomly ordered polygons. This list is represented by a sequence, and for decoding the sequences, we used the technique presented in [19]. To avoid overlapping, the algorithm uses no-fit raster, concept also introduced in [19]. In no-fit raster, to represent the strip a discrete grid of points is used. The scale used for discretization in most instances is 1.0, except for Albano, Dagli, and Swim, (instances in which the area occupied by the polygons is greater), which are $0.02,0.5$, and 0.00005 , respectively. Although in the model we allow free rotation, this bottomleft algorithm does not allow free rotation of the polygons, therefore we use four predefined angles of rotation $\left(0^{\circ}, 90^{\circ}, 180^{\circ}\right.$ and $\left.270^{\circ}\right)$ in all instances. For each instance, we execute the algorithm 1000 times, then we choose the layout with shortest length obtained from these 1000 executions as a starting solution.

\subsection{Comparing results}

To test our model, we will use the same benchmark problems used in the two approaches with which we will compare results, available in [11]. The most important characteristics of these instances are presented in Table 1. The names of the instances are presented in the first column. In the second and third columns the number of convex, and nonconvex polygons are presented, respectively. The total number of polygons, after decomposition of nonconvex polygons into 
convex polygons are presented in the fourth column. In the fifth the total number of vertices are presented. The number of variables and the number of constraints are presented in the sixth and seventh columns, respectively.

Table 1 - Instances data.

\begin{tabular}{c|r|r|r|r|r|r}
\hline Instance & $\begin{array}{c}\text { convex } \\
\text { polygons }\end{array}$ & $\begin{array}{c}\text { nonconvex } \\
\text { polygons }\end{array}$ & $\begin{array}{c}\text { final conv. } \\
\text { poly. no. }\end{array}$ & $\begin{array}{c}\text { no. of } \\
\text { vertices }\end{array}$ & $\begin{array}{c}\text { no. of } \\
\text { variables }\end{array}$ & $\begin{array}{c}\text { no. of } \\
\text { constraints }\end{array}$ \\
\hline albano & 10 & 14 & 52 & 220 & 3907 & 11700 \\
blaz & 16 & 12 & 48 & 216 & 3385 & 10792 \\
dagli & 21 & 9 & 51 & 228 & 3790 & 11988 \\
jakobs1 & 15 & 10 & 35 & 146 & 1831 & 5468 \\
jakobs2 & 14 & 11 & 42 & 158 & 2590 & 7367 \\
marques & 10 & 14 & 50 & 214 & 3628 & 11002 \\
poly1a & 10 & 5 & 22 & 81 & 712 & 1968 \\
poly2a & 20 & 10 & 44 & 162 & 2875 & 7500 \\
poly3a & 30 & 15 & 66 & 243 & 6490 & 16596 \\
poly4a & 40 & 20 & 88 & 324 & 11557 & 29256 \\
poly5a & 50 & 25 & 110 & 405 & 18076 & 45480 \\
poly10a & 100 & 50 & 220 & 810 & 72451 & 180060 \\
poly20a & 200 & 100 & 440 & 1620 & 290101 & 716520 \\
shirts & 60 & 39 & 169 & 739 & 42583 & 126236 \\
swim & 6 & 42 & 291 & 1446 & 123787 & 415845 \\
trousers & 48 & 16 & 104 & 468 & 16045 & 49476 \\
\hline
\end{tabular}

A starting solution is the layout with shortest length, among 1000 executions of the bottom-left algorithm applied to random piece sequences. As the sequence of the polygons is random, if we execute the algorithm another 1000 times, the layout with shortest length can be different to the one found previously, thus, in order to analyze the effectiveness of our model, for each instance, we considered 10 starting solutions. We execute IPOPT to solve our model with each one of these 10 different starting solutions.

When in the initial layout the polygons have enough space to move, the model is able to change the layout more deeply. On the other hand, when the initial layout is already quite compressed, the model performs a compaction phase, without deep changes in the layout. In order to show how much our model reduces the length of the starting solution, we present Table 2, in which the compaction percentages, measured by the layout length reduction from the initial to the final solution, are presented (first column); the minimum, the average and the maximum compaction percentages, regarding the 10 different starting solutions used, are also presented in the third, fourth and fifth columns, respectively. Note that, these percentages are larger in instances with pieces of similar sizes, like in all variations of the instance poly, and that for instances with a smaller number of pieces the compaction is bigger. Note also that the compaction is smaller in instances with big pieces, such as in the instances albano, marques, shirts or trousers. 
Table 2 - Compaction percentages from length in the starting solution to length in the solution.

\begin{tabular}{c|r|r|r|r}
\hline Instance & Compc. & Min. Compc. & Avg. Compc. & Max. Compc. \\
\hline albano & $2.60 \%$ & $0.00 \%$ & $2.20 \%$ & $4.55 \%$ \\
blaz & $4.06 \%$ & $1.80 \%$ & $6.27 \%$ & $11.84 \%$ \\
dagli & $8.17 \%$ & $2.50 \%$ & $5.07 \%$ & $8.17 \%$ \\
jakobs1 & $13.33 \%$ & $0.00 \%$ & $4.00 \%$ & $13.33 \%$ \\
jakobs2 & $7.14 \%$ & $0.00 \%$ & $5.26 \%$ & $8.90 \%$ \\
marques & $3.80 \%$ & $0.00 \%$ & $1.58 \%$ & $3.80 \%$ \\
poly1a & $26.23 \%$ & $15.90 \%$ & $20.71 \%$ & $26.89 \%$ \\
poly2a & $20.71 \%$ & $11.25 \%$ & $16.75 \%$ & $20.71 \%$ \\
poly3a & $18.71 \%$ & $7.29 \%$ & $15.08 \%$ & $18.71 \%$ \\
poly4a & $17.75 \%$ & $13.34 \%$ & $15.68 \%$ & $17.99 \%$ \\
poly5a & $16.86 \%$ & $13.21 \%$ & $15.46 \%$ & $16.98 \%$ \\
poly10a & $15.78 \%$ & $7.27 \%$ & $14.46 \%$ & $15.97 \%$ \\
poly20a & $14.18 \%$ & $2.05 \%$ & $10.38 \%$ & $14.24 \%$ \\
shirts & $2.81 \%$ & $0.56 \%$ & $2.10 \%$ & $3.70 \%$ \\
swim & $9.41 \%$ & $4.30 \%$ & $6.74 \%$ & $10.09 \%$ \\
trousers & $2.59 \%$ & $1.24 \%$ & $2.69 \%$ & $4.15 \%$ \\
\hline
\end{tabular}

The length of the starting solution does not have a determinant impact on the length of the solution, the layout of the starting solution is what determines the length of the solution; two starting solutions with the same length can produce two solutions with quite different lengths, as long as the layouts at the starting solutions are enough different, see Figure 3. In our experiments, we have observed that in 10 of the 16 instances tested the shortest starting solution produced the shortest solution.

We solved the model in Section 2.3 for the instances of Table 1 using the 10 different starting solutions and we compared our results with those of the recent literature that allow free rotations, [29] and [16]. These results are summarized in Table 3, in which the minimum (second column), the average (third column), and the maximum (fourth column) strip length obtained are presented. The average time used to construct the starting solutions (fifth column) and the average time (sixth column) that was spent to solve the instances are also presented in Table 3 ; the strip length and time reported in [29] are in seventh and eighth columns, and strip length and time reported in [16] are in ninth and tenth columns, respectively.

For those instances tested in [29], it can be observed that the length of the best solution obtained in this work is slightly greater than those reported there. However, when comparing with [16] we can see that in most instances the minimum length obtained using our model is smaller. In the few remaining, the length is very close. On the other hand, note that our model finds good solutions for problems with a large number of polygons, although in these the number of variables and constraints, and the computational time, grows drastically. These solutions may not be local optimal due to the maximum execution time. 


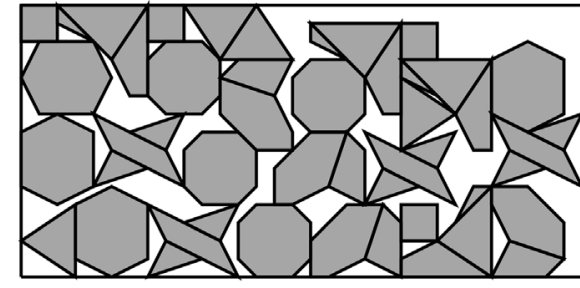

(a) Starting solution layout 1

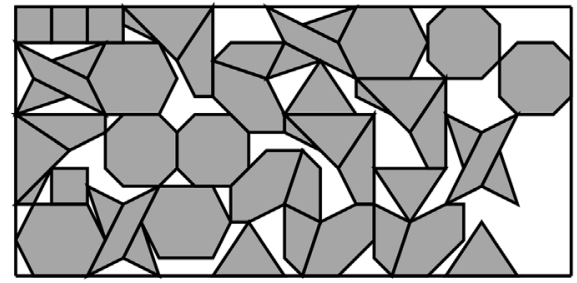

(c) Starting solution layout 2

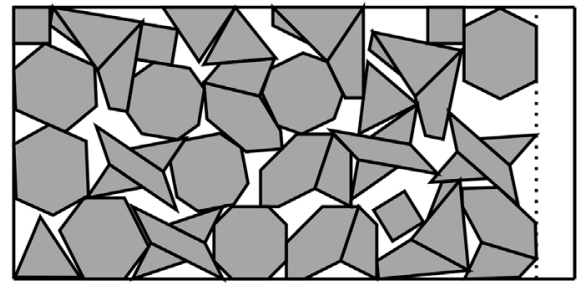

(b) Final layout 1

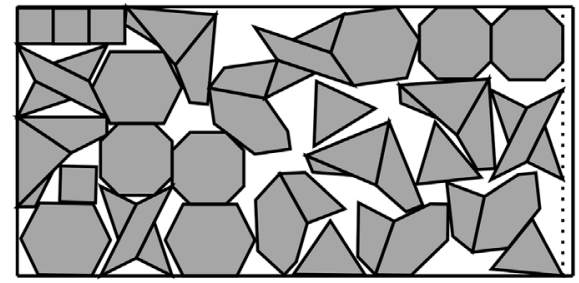

(d) Final layout 2

Figure 3 - Example of two starting solutions with the same length and solutions with quite different lengths.

Table 3 - Comparison of our results to those in [29] and [16].

\begin{tabular}{|c|c|c|c|c|c|c|c|c|c|}
\hline \multirow[b]{2}{*}{ Instance } & \multicolumn{5}{|c|}{ Our approach } & \multicolumn{2}{|c|}{ Best results in [29] } & \multicolumn{2}{|c|}{ Best results in [16] } \\
\hline & $\begin{array}{l}\text { Minimal } \\
\text { Solution } \\
\end{array}$ & $\begin{array}{l}\text { Average } \\
\text { Solution } \\
\end{array}$ & $\begin{array}{l}\text { Maximal } \\
\text { Solution } \\
\end{array}$ & $\begin{array}{l}\text { SP Average } \\
\text { time(sec) }\end{array}$ & $\begin{array}{l}\text { IPOPT Average } \\
\text { time(sec) }\end{array}$ & Length & Time(sec) & Length & Time(sec) \\
\hline albano & 10355.80 & 10601.03 & 10849.99 & 158.68 & 178.56 & & & 10032.24 & 124.39 \\
\hline blaz & 27.82 & 29.02 & 30.44 & 1.37 & 49.52 & 25.41 & 25.42 & 28.27 & 56.86 \\
\hline jakobs1 & 12.99 & 13.49 & 14.00 & 4.68 & 19.30 & & & 13.19 & 48.46 \\
\hline jakobs2 & 26.00 & 27.29 & 30.00 & 17.60 & 18.41 & & & 24.25 & 53.67 \\
\hline marques & 84.65 & 86.68 & 91.00 & 66.74 & 58.11 & & & 84.93 & 118.12 \\
\hline polyla & 14.01 & 14.53 & 15.01 & 6.23 & 19.07 & & & 13.90 & 27.59 \\
\hline poly4a & 50.99 & 52.50 & 53.72 & 20.59 & 1621.54 & & & 51.13 & 210.74 \\
\hline poly5a & 63.66 & 65.05 & 66.31 & 26.21 & 1773.85 & & & 65.64 & 287.32 \\
\hline poly $10 a$ & 127.16 & 129.46 & 140.01 & 58.25 & 2350.17 & 126.29 & 618.80 & & \\
\hline poly20a & 254.86 & 268.06 & 294.81 & 151.38 & 3484.92 & 251.04 & 1209.17 & & \\
\hline shirts & 62.19 & 64.43 & 65.62 & 11.55 & 1808.73 & & & 65.06 & 340.89 \\
\hline swim & 6011.93 & 6311.16 & 6526.38 & 449.85 & 3600.00 & 5661.95 & 431.97 & & \\
\hline trousers & 249.35 & 258.03 & 261.94 & 118.00 & 603.36 & & & 251.94 & 265.48 \\
\hline
\end{tabular}

The layout of minimum length obtained and the starting solution used to find it, for each instance, can be seen in Figures $4-19$. 


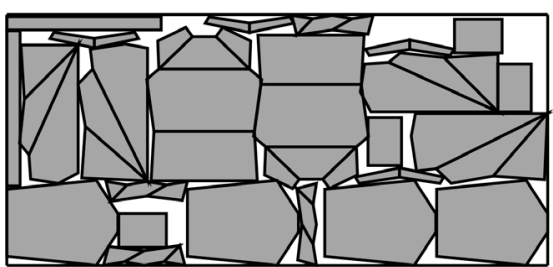

(a) Starting solution layout

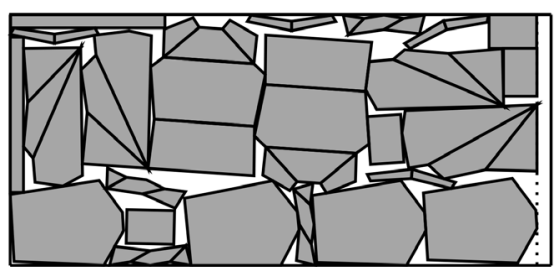

(b) Final layout

Figure 4 - Instance Albano.

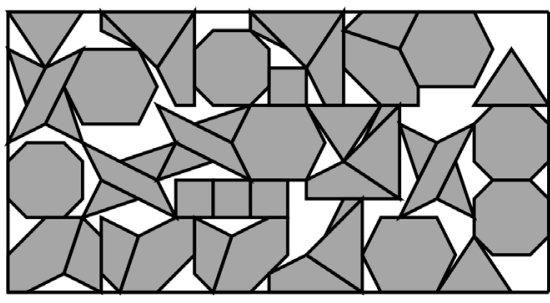

(a) Starting solution layout

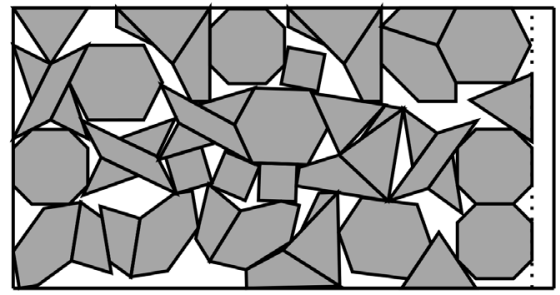

(b) Final layout

Figure 5 - Instance Blaz.

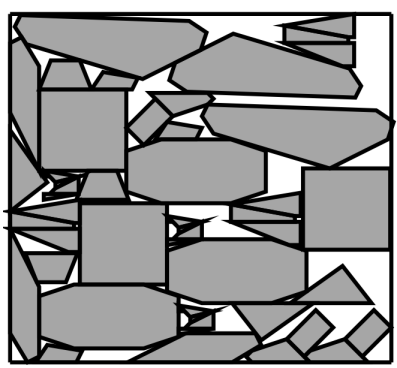

(a) Starting solution layout

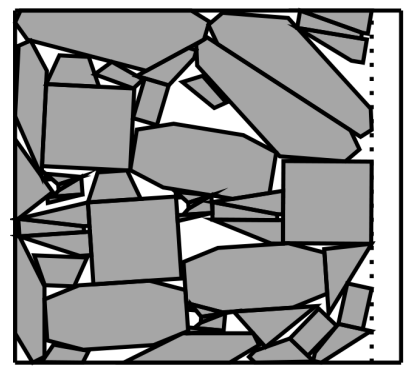

(b) Final layout

Figure 6 - Instance Dagli.

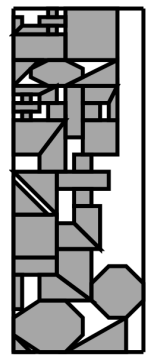

(a) Starting solution layout

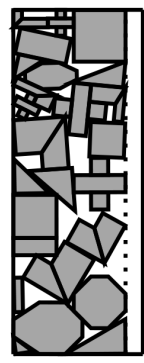

(b) Final layout

Figure 7 - Instance Jakobs1. 


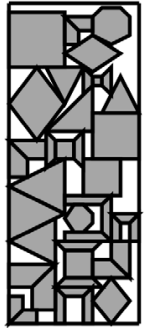

(a) Starting solution layout

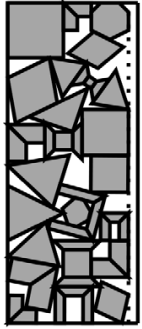

(b) Final layout

Figure 8 - Instance Jakobs2.

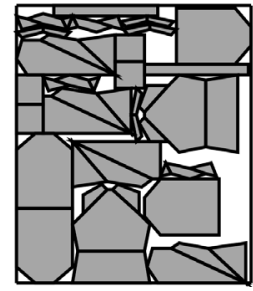

(a) Starting solution layout

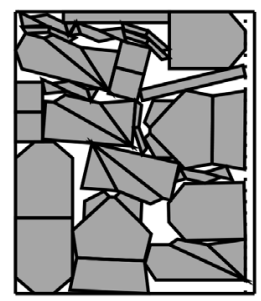

(b) Final layout

Figure 9 - Instance Marques.

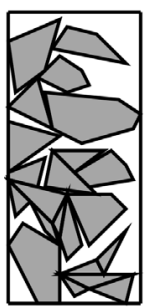

(a) Starting solution layout

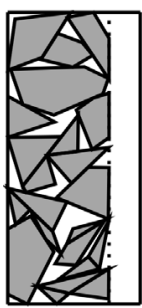

(b) Final layout

Figure 10 - Instance Poly1a.

\section{CONCLUSIONS}

In this work, we developed a model for irregular strip packing problems, that allows free rotations and uses separation lines to avoid overlap. As a relevant point of this work we highlight the use of the general equation of the line to model the separation lines, allowing us to use only three variables for each separation line: the coordinates of the reference point and the angle of rotation. This establishes a marked dissimilarity with other models presented in the literature, for example the one presented in [15], which has a higher number of variables. The number of variables in the model used in [15] is grounded on the representation of the polygons used, in addition to the 


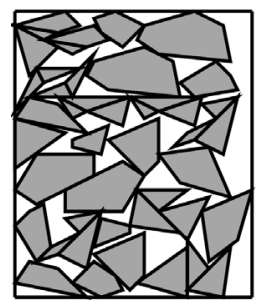

(a) Starting solution layout

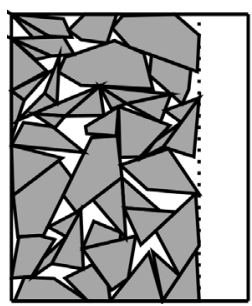

(b) Final layout

Figure 11 - Instance Poly2a.

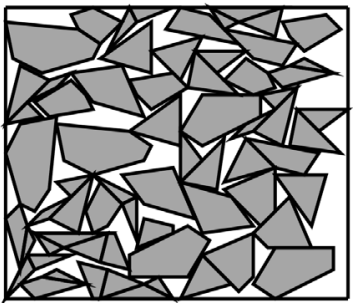

(a) Starting solution layout

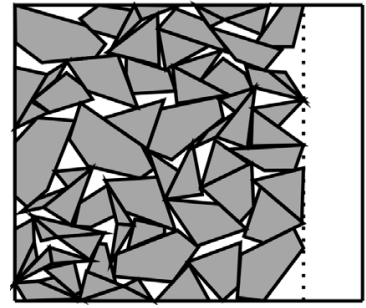

(b) Final layout

Figure 12 - Instance Poly3a.

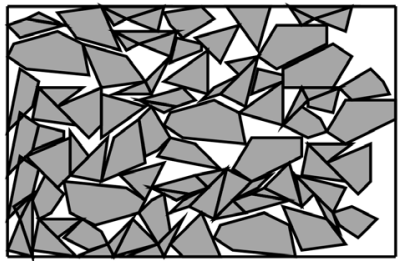

(a) Starting solution layout

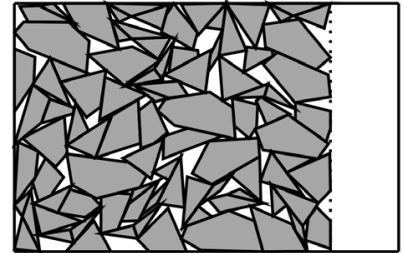

(b) Final layout

Figure 13 - Instance Poly4a.

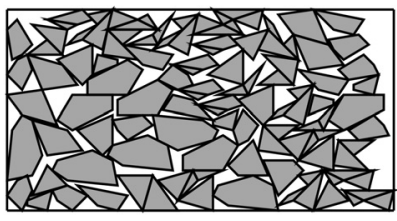

(a) Starting solution layout

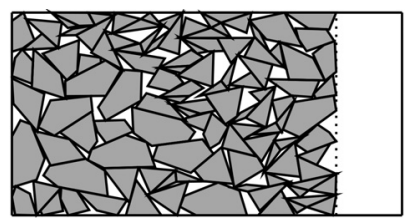

(b) Final layout

Figure 14 - Instance Poly5a. 


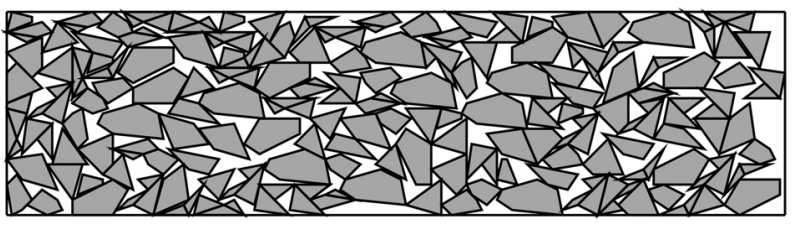

(a) Starting solution layout

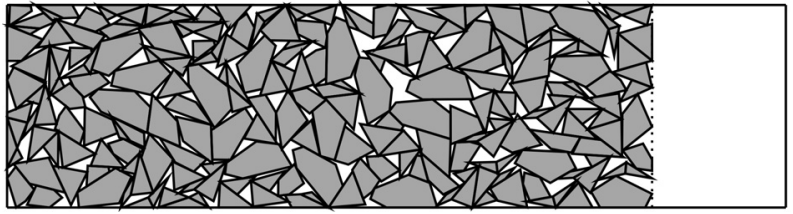

(b) Final layout

Figure 15 - Instance Poly10a.

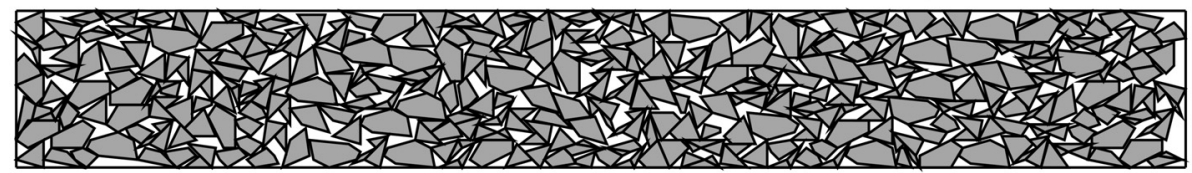

(a) Starting solution layout

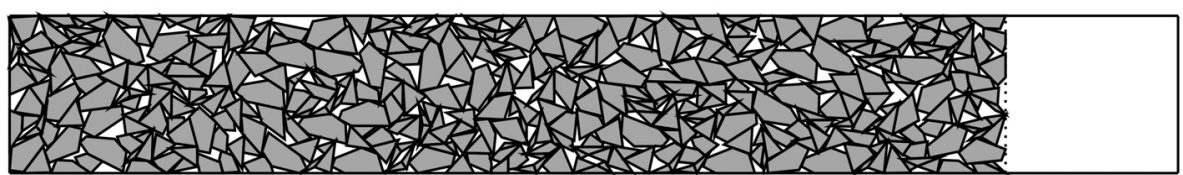

(b) Final layout

Figure 16 - Instance Poly20a.

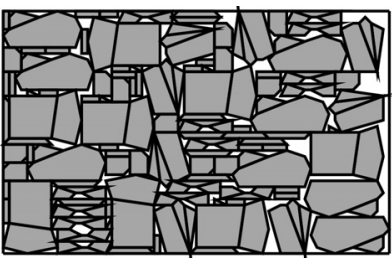

(a) Starting solution layout

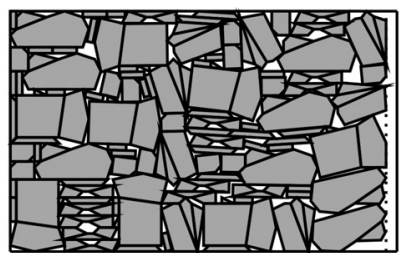

(b) Final layout

Figure 17 - Instance Shirts. 


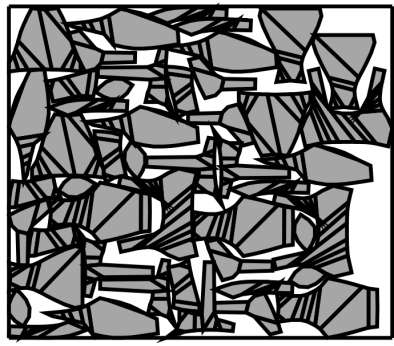

(a) Starting solution layout

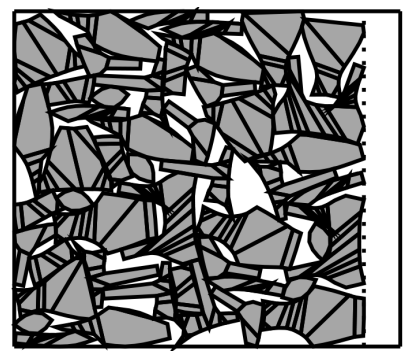

(b) Final layout

Figure 18 - Instance Swim.

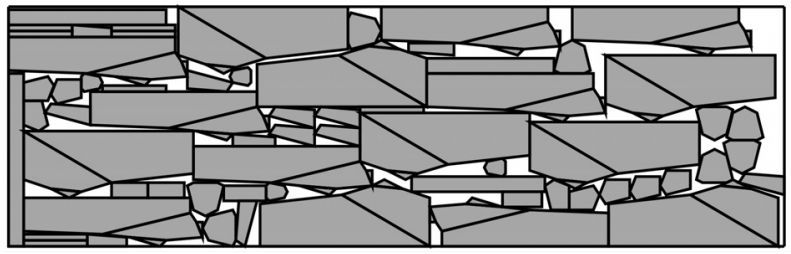

(a) Starting solution layout

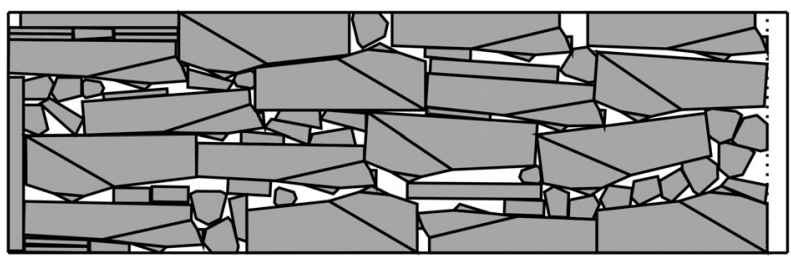

(b) Final layout

Figure 19 - Instance Trousers.

vector equation of the line used to model the separation line. In fact, a instance with two convex polygons of 5 and 6 vertices would use:

- For the separation lines: eleven variables for the distances of the vertices to the separation line, two variables for the footing point vector, twenty two variables for the vectors connecting the separation line with the vertices, eleven auxiliary variables needed to compute these vectors, two variables for the direction vector and, two variables for the normal vector.

- For the polygons: two variables for the orientation angle of the polygons, two variables for the radius of the smallest circle enclosing the polygons, twenty two variables for the coordinates of the vertices and, four variables for the center coordinates of the polygons.

- Others: a variable for the area of the design rectangle, two variables for the extensions of the design rectangle and, a variable for the objective function. 
Instead, for an instance with two convex polygons our model would only need 10 variables, a significant simplification that leads to a better performance of the solution method ensuring likewise a good solution to the problem.

The solution of the problem modeled here, using a local nonlinear programming solver, depends on the starting solution. We used a bottom-left algorithm to construct these starting solutions. To test the effectiveness of our model, we compared our results with those obtained recently in the literature $[16,29]$, which also use methodologies with free rotations. The lengths reported in [29] are smaller but very close to those found in this work. On the other hand, the lengths reported in [16] are greater in most instances; in the others, they are very close. Therefore, the effectiveness of our model is verified, as well as the quality of the constructed starting solutions using a bottom-left algorithm; however, we believe that these results could be improved by using another algorithm to construct the starting solutions.

\section{ACKNOWLEDGEMENTS}

This research was partially supported by CNPq (grants 141072/2014-8 and 409043/2016-8) and FAPESP (grants 2013/07375-0 and 2016/01860-1), from Brazil.

\section{REFERENCES}

[1] Albano A \& Sapuppo A. 1980. Optimal allocation of two-dimensional irregular shapes using heuristic search methods. IEEE Transactions on Systems, Man and Cybernetics, 10: 242-248.

[2] Alvarez-Valdes R, Martinez A \& Tamarit JM. 2013. A branch and bound algorithm for cutting and packing irregularly shaped pieces. International Journal of Production Economics, 145(2): 463-477.

[3] Andreani R, Birgin EG, Martinez JM \& Schuverdt ML. 2007. On augmented lagrangian methods with general lower-level constraints. SIAM Journal on Optimization, 18: 1286-1309.

[4] Andreani R, Birgin EG, Martinez JM \& Schuverdt ML. 2008. Augmented lagrangian methods under the Constant Positive Linear Dependence constraint qualification. Mathematical Programming, 111: 5-32.

[5] Bennell JA \& Oliveira JF. 2008. The geometry of nesting problems: A tutorial. European Journal of Operational Research, 184: 397-415.

[6] Bennell JA, Scheithauer G, Stoyan Y \& Romanova T. 2010. Tools of mathematical modelling of arbitrary object packing problems. Ann. Oper. Res., 179: 343-368.

[7] CGAl - Computational Geometry Algorithms Library. 2D Polygon Partitioning. Available at: http://doc.cgal.org/latest/Partition_2/

[8] Chernov N, Stoyan Y \& Romanova T. 2010. Mathematical model and efficient algorithms for object packing problem. Computational Geometry: Theory and Applications, 43: 535-553.

[9] Cherri L, Carravilla M \& Toledo F. 2016. A model-based heuristic for the irregular strip packing problem. Pesquisa Operacional, 36(3): 447-468. 
[10] Egeblad J, Nielsen BK \& OdgaARd A. 2007. Fast neighborhood search for two and threedimensional nesting problems. European Journal of Operational Research, 183: 1294-1266.

[11] EURO Special Interest Group on Cutting And Packing. 2015. Available at: http:// paginas.fe.up.pt/ esicup/datasets

[12] FischetTi M \& LUZZI I. 2009. Mixed-integer programming models for nesting problems. Journal of Heuristics, 15(3): 201-226.

[13] Gomes AM \& Oliveira JF. 2002. A 2-exchange heuristic for nesting problems. European Journal of Operational Research, 141: 359-370.

[14] JONES DR. 2013. A fully general, exact algorithm for nesting irregular shapes. Journal of Global Optimization, 59: 367-404.

[15] KallRath J. 2009. Cutting circles and polygons from area-minimizing rectangles. Journal of Global Optimization, 43: 299-328.

[16] Liao X, Ma J, OU C, Long F \& LiU X. 2016. Visual nesting system for irregular cutting-stock problem based on rubber band packing algorithm. Advances in Mechanical Engineering, 8(6): 1-15.

[17] Marques VM, Bispo CF \& Sentieiro JJ. 1991. A system for the compaction of two-dimensional irregular shapes based on simulated annealing. IEEE Transactions on Industrial Electronics, Control and Instrumentation, 3: 1911-1916.

[18] Misener R \& Floudas CA. 2013. GloMIQO: global mixed-integer quadratic optimizer. Journal Global Optimization, 57: 3-50.

[19] Mundim LR, Andretta M \& Queiroz TA. 2017. A biased random key genetic algorithm for open dimension nesting problems using no-fit raster. Expert Systems with Applications, 81: 358-371.

[20] Nielsen BK. 2007. An efficient solution method for relaxed variants of the nesting problem. Proceedings of the thirteenth Australasian symposium on Theory of computing, 65: 123-130.

[21] Nocedal J, W Ächter A \& WAltz RA. 2009. Adaptive barrier strategies for nonlinear interior methods. SIAM Journal on Optimization, 19: 1674-1693.

[22] Oliveira JF \& Ferreira JS. 1993. Algorithms for nesting problems Applied simulated annealing, In: VIDAL RVV. (Ed.), Lecture notes in econ. and Maths Systems. Springer Verlag, 396: 255-274.

[23] Rocha P, Rodrigues R, Gomes AM, Toledo FMB \& Andretta M. 2015. Two-Phase Approach to the Nesting problem with continuous rotations. IFAC-PapersOnline, 48(3): 501-506.

[24] SAHINIDIs NV. 2014. BARON 14.3.1: Global Optimization of Mixed-Integer Nonlinear Programs. User's Manual. Available at: http: / /www.minlp.com/downloads / docs /baron\ ○20manual.pdf

[25] Segenreich SA \& Braga LM. 1986. Optimal nesting of general plane figures: a Monte Carlo heuristical approach. Computers and Graphics, 10: 229-237.

[26] Stoyan YG, Terno J, Scheithauer G, Gil N \& Romanova T. 2001. Phi-functions for primary 2d-objects. Studia Informatica Universalis, 2(1): 1-32.

[27] Stoyan YG, Scheithauer G, Gil N \& Romanova T. 2004. Phi-functions for complex 2dobjects. 4OR: Quartely Journal of the Belgian, French and Italian Operations Research Societies, 2 : 69-84. 
[28] Stoyan YG \& Chugay AM. 2008. Packing cylinders and rectangular parallelepipeds with distances between them. European Journal Operation Research, 197: 446-455.

[29] Stoyan YG, Pankratov A \& Romanova T. 2016. Cutting and packing problems for irregular objects with continuous rotations: mathematical modelling and non-linear optimization. Journal of the Operational Research Society, 67(5): 786-800.

[30] Tawarmalani M \& SAhinidis NV. 2005. A polyhedral brach-and-cut approach to global optimization. Mathematical Programming, 103(2): 225-249.

[31] Toledo FMB, Carravilla MA, Ribeiro C, Oliveira JF \& Gomes AM. 2013. The dottedboard model: A new mip model for nesting irregular shapes. International Journal of Production Economics, 145(2): 478-487.

[32] WëChter A \& BIEgleR LT. 2006. On the implementation of a primal-dual interior point filter line search algorithm for large-scale nonlinear programming. Mathematical Programming, 106(1): $25-57$.

[33] Wëchter A \& Biegler LT. 2015. COIN OR project, Available at: http://projects. coin-or.org/Ipopt

[34] Zoutendisk G. 1960. Methods of feasible directions, a study in linear and non-linear programming, Elsevier.

[35] Zoutendisk G. 1970. Nonlinear programming, computational methods. Integer and Nonlinear Programming, 143(1): 37-86. 